\title{
Review of: "Highly conductive electronics circuits from aerosol jet printed silver inks"
}

Yi Fang ${ }^{1}$

1 Beijing Institute Of Graphic Communication

Potential competing interests: The author(s) declared that no potential competing interests exist.

This paper presented the preparation and printing of silver NP Inks with the addition of several types of surfactant with diferent concentrations-0, 0.5, 1, 1.5, 2 wt.\%. Printed patterns were tested to defne which surfactant concentration and type result in the lowest values of electrical resistivity. Resistivity of $4.5 \cdot 10-8$ $\Omega m$ was achieved. Generally speaking, the paper has certain application value. However, due to the characteristics of aerosol jet, its accuracy is limited, which limits its application. In addition, I am afraid there is a mistake in the caption of Figure 5. I think (a) was the the line height and b) was the line width. 